\title{
Omnidirectional vision on UAV for attitude computation
}

\author{
Cédric Demonceaux, Pascal Vasseur and Claude Pégard \\ C.R.E.A. (Centre de Robotique, d'Electrotechnique et d'Automatique) - EA 3299 \\ Université de Picardie Jules Verne \\ 7, Rue du Moulin Neuf \\ 80000 Amiens \\ Email: \{Cedric.Demonceaux, Pascal.Vasseur, Claude.Pegard $\} @$ u-picardie.fr
}

\begin{abstract}
Unmanned Aerial Vehicles (UAVs) are the subject of an increasing interest in many applications. Autonomy is one of the major advantages of these vehicles. It is then necessary to develop particular sensors in order to provide efficient navigation functions. In this paper, we propose a method for attitude computation catadioptric images. We first demonstrate the advantages of the catadioptric vision sensor for this application. In fact, the geometric properties of the sensor permit to compute easily the roll and pitch angles. The method consists in separating the sky from the earth in order to detect the horizon. We propose an adaptation of the Markov Random Fields for catadioptric images for this segmentation. The second step consists in estimating the parameters of the horizon line thanks to a robust estimation algorithm. We also present the angle estimation algorithm and finally, we show experimental results on synthetic and real images captured from an airplane.
\end{abstract}

\section{INTRODUCTION}

Unmanned Aerial Vehicles (UAV) have proved their usefulness in many applications. However, their autonomy requires a lot of abilities such as being able to evaluate their global position, their altitude or their attitude in order to control their stability. In this work, we propose to use a central catadioptric vision sensor in order to compute the attitude of a UAV. With this system, we are able to evaluate the roll $(\rho)$ and pitch $(\psi)$ angles without any additional inertial or rate sensors. The idea consists in using the particular geometric characteristics of the catadioptric sensor in order to compute precisely these angles.

Generally, attitude computation by vision is based on the detection of the horizon in the image and on the estimation of the angle between the horizon and a horizontal reference line. In [1], the authors use a perspective camera and they consider the horizon as a straight line. The roll angle is then given by the inverse tangent of the slope of the horizon line and the pitch angle is approximated by the percentage of sky in the image. Then, they are looking for the horizon line in the image which maximizes an optimization criterion which tends to separate the earth from the sky. In [2], an improved version of the previous algorithm which better manages the variation of the appearance of the sky is presented. In this way, they propose to integrate a texture representation in order to improve the first model which only relies on color information. A statistical framework with prior models of the sky and the earth is also used for the adaptation of the algorithm to the variations of appearance.

In spite of the interesting results obtained with these approaches, the use of a single perspective camera generates several drawbacks. First, we only obtain a partial view of the environment and important occlusions in the horizon can have a serious influence on the final result. Second, the horizon is visible only in a particular interval of roll and pitch values. If the UAV gets out of this interval, the final image is exclusively made of sky or earth and the horizon can not be detected. Third, with a perspective camera, it is only possible to compute the roll angle while the pitch is only approximated thanks to a hypothesis on the altitude of the UAV. In this work, we propose to use an omnidirectional visual sensor in order to compute the attitude of a UAV. This kind of sensor has already been used for the navigation of UAVs as in [3] and omnidirectional vision has proved its usefulness in a lot of robotic applications [4]. Omnidirectional vision and more particularly catadioptric vision consists in associating a convex mirror with a projective camera whose optical axis is aligned with the axis of the mirror [4]. The main advantage of these sensors is the acquisition of an omnidirectional image with a single shot. In our application of UAV attitude computation, omnidirectional vision presents the following advantages: first, a complete surrounding of the UAV can be captured and the horizon is totally visible. The possible occlusions will then have a lower impact on the estimation of the final results. Second, whatever the attitude of the UAV, the horizon is always present in the image, even partially, and the angles can always be computed. Third, we are able to compute the roll angle but also the pitch angle without any prior hypothesis, contrary to the applications including a perspective camera.

However, catadioptric vision also presents some drawbacks. For example, a catadioptric image contains significant deformations due to the geometry of the mirror and to the sampling of the camera [5] and these deformations have important consequences for the image processing. So, direct application of classical operators can not provide satisfactory results. We then also show in this paper an adaptation of the Markov Random Fields (MRFs) which permits to treat the images while taking into account these deformations. 


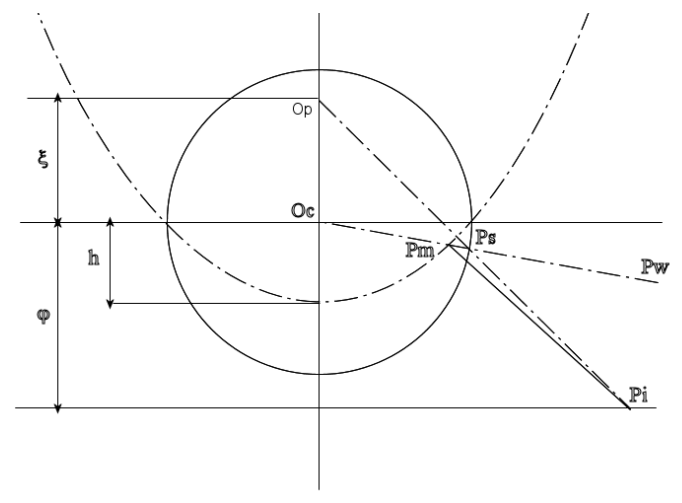

Fig. 1. Equivalence between the catadioptric projection and the two-step mapping via the sphere.

We use this adaptation in order to detect the horizon in the catadioptric image. Once this horizon line is detected, we use the particular geometric characteristics of the catadioptric sensor in order to compute the attitude of the UAV.

In the rest of the paper, we present in Section 2 the model of image formation and how the horizon is projected in the catadioptric image. Section 3 is devoted to the adaptation of MRFs to the catadioptric images and to its implementation for the ground/sky segmentation. In Section 4, we develop the estimation of the parameters of the horizon and consequently of the roll and pitch angles. Experimental results and a discussion are proposed in Section 5. Finally, we conclude in Section 6 with a summary and perspectives.

\section{IMAGE Formation AND HORIZON PROJECTION}

\section{A. Central Catadioptric Image Formation}

Baker and Nayar classified catadioptric sensors into two categories depending on the number of viewpoints [6]. Sensors with a single viewpoint, named central catadioptric sensors, permit a geometrically correct reconstruction of the perspective image from the original catadioptric image. This category is made of a parabolic mirror associated to an orthographic camera and hyperbolic, elliptic and plane mirrors with a perspective camera. The second category with several viewpoints has much less significant geometric properties and is made of the other possibilities of associations between mirrors and cameras. In this paper, we are only interested in central sensors, for which Geyer and Daniilidis have demonstrated the equivalence with a two-step projection via a unitary sphere centered on the focus of the mirror (the single viewpoint) [7]. This two-step projection first consists in projecting a 3D point to the sphere from the center of the sphere. The next step consists in projecting the point on the sphere to the image plane from a point $O_{p}$ placed on the optical axis (Fig 1). The position of this point of projection $O_{p}$ and of the image plane are determined with the calibration of the sensor.

\section{B. Projection from the Image to the Sphere}

From the equivalence model developed in [7] and showed in Figure 1, we have :

$$
\begin{aligned}
& x_{s}=\gamma x_{w} \\
& y_{s}=\gamma y_{w} \\
& z_{s}=\gamma z_{w}
\end{aligned}
$$

The equation of the unitary sphere is $x_{s}^{2}+y_{s}^{2}+z_{s}^{2}=1$ and then gives :

$$
\gamma=\frac{1}{\sqrt{x_{w}^{2}+y_{w}^{2}+z_{w}^{2}}}
$$

The coordinates of point $P_{i}$ on the image plane are then obtained from the perspective projection of $P_{s}$ and parameters $(\xi, \varphi)$ of the mirror :

$$
\begin{gathered}
\frac{x_{s}}{\xi-z_{s}}=\frac{x}{\xi+\varphi} \\
\frac{y_{s}}{\xi-z_{s}}=\frac{y}{\xi+\varphi} \\
z=-\varphi
\end{gathered}
$$

where $(x, y, z)$ are the coordinates of point $P_{i}$ expressed in the metric system and $\xi$ and $\varphi$ define the shape of the mirror (see [8] for further details on their signification).

$$
\left\{\begin{array}{c}
x=\frac{(\xi+\varphi) x_{w}}{\xi \sqrt{x_{w}^{2}+y_{w}^{2}+z_{w}^{2}}-z_{w}} \\
y=\frac{(\xi+\varphi) y_{w}}{\xi \sqrt{x_{w}^{2}+y_{w}^{2}+z_{w}^{2}}-z_{w}} \\
z=-\varphi
\end{array}\right.
$$

In the image frame, we finally obtain :

$$
\left(\begin{array}{l}
u \\
v
\end{array}\right)=\left(\begin{array}{ccc}
\alpha_{u} & 0 & u_{0} \\
0 & \alpha_{v} & v_{0}
\end{array}\right)\left(\begin{array}{l}
x \\
y \\
1
\end{array}\right)
$$

where $(u, v)$ are the coordinates of $P_{i}$ in pixels and $\left(\alpha_{u}, \alpha_{v}, u_{0}, v_{0}\right)$ are the intrinsic parameters of the camera. In order to perform the calibration with this model, we place the catadioptric system in a cube with a grid of points on each side. In this way, the points of the pattern are distributed over the whole catadioptric image. The pattern contains 144 points and we estimate six extrinsic parameters (3 rotations and 3 translations): the parameters $\xi$ and $\varphi$ of the mirror as well as the intrinsic parameters of the camera $\left(\alpha_{u}, \alpha_{v}, u_{0}, v_{0}\right)$. The estimation is performed by the minimization of the quadratic error between the selected points and those computed by the model. With this method, we are then able to calibrate any central catadioptric sensor without restriction about the shape of the mirror as in the method proposed in [9].

\section{Central Catadioptric Projection of the Horizon}

As demonstrated in [9], a 3D sphere projects on the equivalent sphere in a small circle, and then on the catadioptric image plane in an ellipse. Consequently, the attitude computation consists in looking for an ellipse in the omnidirectional image or a small circle on the equivalent sphere which corresponds to the horizon. The geometrical properties of the equivalent sphere allow to deduce the roll and pitch angles. Indeed, if we consider Figure 2, we can note that the normal of the projected 
horizon on the sphere, which is also confounded with the line passing through the center of the sphere of equivalence and through the center of the earth represents in fact the attitude of the UAV depending on the position of the optical axis. Then, the computation of the coordinates of the optical axis is sufficient in order to deduce the roll and pitch angles. From the previous considerations, the complete algorithm will then first consists in segmenting the catadioptric image in order to separate the sky from the ground and in extracting the points of the horizon. Next, we back project the points of the horizon on the sphere of equivalence thanks to the parameters obtained with the calibration. Finally, we estimate the best plane which passes through the points of horizon on the sphere and we deduce its normal and consequently the roll $(\rho)$ and pitch $(\psi)$ angles.

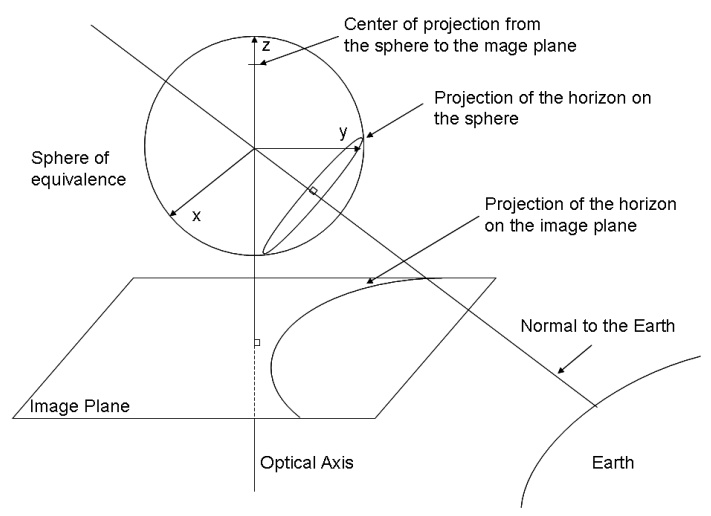

Fig. 2. Relation between the projections of the horizon and the roll $(\rho)$ and pitch $(\psi)$ angles.

\section{Sky/Ground Segmentation In CATAdioptriC IMAGES}

This section deals with the problem of horizon detection. To do this, we choose an MRF modeling in order to segment the omnidirectional image in two parts, one corresponding to the sky and the other one to the ground.

\section{A. Limitation of Classical MRFs in Omnidirectional Images}

Since the eighties and thanks to Besag [10], MRFs have permitted to solve many problems in image processing such as image segmentation, image restoration, motion detection and so on. However, they become unsuitable if they are applied in the same way for catadioptric images. Indeed, MRFs use a local dependence between pixels from a predefined neighborhood, which has to be reconsidered because of the distortions of catadioptric images.

In the case of classical images, the neighboring systems are defined by the closest pixels (generally, neighboring of order 1 or 2). However, this kind of neighborhood is not suited to catadioptric images because of the distortions generated by the mirror. Indeed, a pixel at a distance $d$ of pixel $p$ does not have the same influence on the latter if $p$ is placed either in the center of the image or in its periphery.

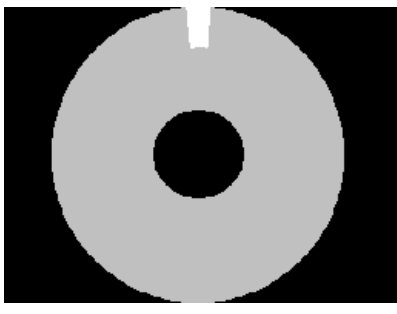

(a)

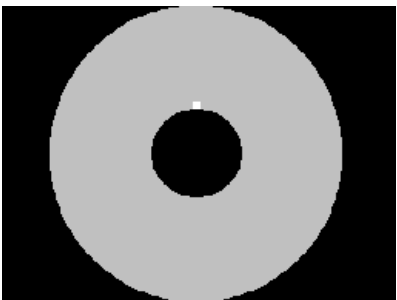

(b)
Fig. 3. (a) Neighborhood at the periphery of the image, (b) Neighborhood in the center of the image.

\section{B. An Adapted Neighborhood}

To find a neighboring more appropriate to an omnidirectional image, we propose to use the equivalence between the catadioptric projection and the sphere (Fig. 1).

In the model of equivalence [7], the mapping function is equivalent to a projection of a $3 \mathrm{D}$ point $P_{w}$ to a point $P_{s}$ on the sphere, followed by a projection from a point on the optical axis to point $P_{i}$ on the image plane (Fig. 1). The center of the sphere is equal to the mirror focus and its radius is function of the latus rectum of the mirror. The position of the center of projection in the second projection depends on the shape and the dimensions of the mirror.

Let us pose $P_{i}$ an omnidirectional image point of polar coordinates $(u, v)=(D \cos (\phi), D \sin (\phi))$ in camera coordinate system which center corresponds to the mirror axis.

The equivalent point $P_{s}$ on the sphere has then as spherical coordinates $(1, \theta, \phi)$ in the coordinate system associated with the equivalent sphere of radius 1 :

$$
\theta=\arccos \left(\frac{\xi D^{2}-(\xi+\varphi) \sqrt{(\xi+\varphi)^{2}+D^{2}\left(1-\xi^{2}\right)}}{D^{2}+(\xi+\varphi)^{2}}\right)
$$

This change of space makes possible to overcome the distortions induced by the sensor. To create a spatial dependence between the points, it thus appears more natural to consider points $P_{s}$ instead of points $P_{i}$ by associating to these points a neighborhood defined on the equivalent sphere.

Let $\Pi$ the projection for which any site $s$ (pixel of the omnidirectional image) associates its equivalent point $P_{s}$ in spherical coordinates on unitary sphere $S^{2}$ (Fig 1),

$$
\Pi: \begin{array}{llc}
\mathcal{S} & \rightarrow & S^{2} \\
s=(u, v) & \mapsto & \left(1, \theta_{s}, \phi_{s}\right)
\end{array}
$$

Then we define the new neighborhood as follows (Fig 3):

$$
\begin{aligned}
& \forall s \in \mathcal{S}, \Pi(s)=\left(1, \theta_{s}, \phi_{s}\right) \\
& \mathcal{V}_{s}=\left\{\begin{array}{l}
t \in \mathcal{S}, t \neq s \quad \Pi(t)=\left(1, \theta_{t}, \phi_{t}\right) \text { such as } \\
\left|\theta_{t}-\theta_{s}\right| \leq \frac{1}{N} \text { and } \\
\left(\left|\phi_{t}-\phi_{s}\right| \leq \frac{1}{M} \text { or }\left|\phi_{t}-\phi_{s}\right| \geq 2 \pi-\frac{1}{M}\right)
\end{array}\right\},
\end{aligned}
$$

where $N$ and $M$ are two fixed constants which define the order of the neighborhood.

Let us note that we do not carry out any sampling on the 
equivalent sphere. Indeed, the new neighborhood is defined only starting from the points of the sphere corresponding to pixels of the omnidirectional image.

We have shown in [11] that this neighborhood is more appropriate for omnidirectional images than a classical neighborhood used for perspective images.

\section{Markovian Formulation of Sky/Ground Segmentation}

We want to divide the image into two groups, one which corresponds to the sky and the other one to the ground in omnidirectional image sequence $I(t)$. To achieve this, we define a Markovian modeling as follows. Let us pose $E(t)=$ $\left\{e_{s}(t), s \in \mathcal{S}\right\}$ the label field where $e_{s}$ can take two values $\{0,1\}(0=$ site of the sky, $1=$ site of the ground), $O(t)=$ $\left\{o_{s}(t), s \in \mathcal{S}\right\}$ is the observation field and where $\mathcal{S}$ is the set of pixels in the image. At each site $s$, observation $o_{s}$ is supplied by color component $c(s)=\left(c_{1}(s), c_{2}(s), c_{3}(s)\right)$ expressed in the space described in [12]:

$$
c_{1}=r-g, \quad c_{2}=2 b-r-g, \quad c_{3}=r+g+b,
$$

with $(r, g, b)$ the color space coordinates in the (red,green,blue) color space. The data-driven potential $q$ at each site is defined by:

$$
q\left(e_{s}, o_{s}\right)=\left(c(s)-M_{e_{s}}\right)^{t} \Sigma_{e_{s}}^{-1}\left(c(s)-M_{e_{s}}\right)
$$

the two regions being modeled by a Gaussian model $\left(M_{e_{s}}, \Sigma_{e_{s}}\right)$. Then, penalization term $v$ which favors the spatial homogeneity is :

$$
v\left(e_{s}, e_{r}\right)=\mu\left(1-\delta\left(e_{s}-e_{r}\right)\right)
$$

where $\mu$ is a positive constant and $\delta$ is the Kronecker symbol.

Let us note $\mathcal{C}_{s r}=\{c \in \mathcal{C} \mid s \in c$ et $r \in c\}$, the maximum a posteriori (MAP) criterion of our modelization is the minimum of:

$$
U(e, o)=\sum_{s \in \mathcal{S}} q\left(e_{s}, o_{s}\right)+\sum_{<s, r>\in \mathcal{C}} \frac{1}{\operatorname{card}\left(\mathcal{C}_{s r}\right)} v\left(e_{s}, e_{r}\right)
$$

where $\mathcal{C}$ are the cliques defined using neighborhood $\mathcal{V}$ (formula (8)).

At time $t=0$, the two Gaussian models defining the Sky and the Ground are evaluated using a disc at the periphery on the omnidirectional image for the sky and a disc at the image center for the ground. Then, the Gaussian models are estimated using the label field of the previous estimation $e(t-1)$.

\section{Horizon Estimation And Attitude Computation}

Once the horizon has been detected in the image, we want to compute the normal of the plane which contains the projection of the horizon on the sphere. This method then first consists in using the results of the calibration in order to project the points from the image to the sphere. Next, we propose to estimate the small circle parameters thanks to a robust M-estimator of Tukey [13] and finally we obtain the roll and pitch angles from the coordinates of the normal of the small circle.

The aim of this step consists in estimating the plane which passes through the points of the horizon on the sphere. Let us consider $N$ horizon points projected on the sphere with coordinates $\left(x_{s}^{i}, y_{s}^{i}, z_{s}^{i}\right)$ with $i=1, \ldots, N$. In order to avoid the outlying points, we need to determine $\Theta=(A, B, C)^{T}$ as the solution of :

$$
\begin{aligned}
\widehat{\Theta} & =\arg \min _{\Theta} \sum_{i} \Gamma\left(r_{i}\right) \\
r^{i} & =A x_{s}^{i}+B y_{s}^{i}+C+z_{s}^{i} .
\end{aligned}
$$

where $\Gamma$ is the robust M-estimator of Tukey's biweight [14]. Solution $\widehat{\Theta}$ of (12) is computed by an Iteratively Reweighted Least Squares (IRLS):

$$
\widehat{\Theta}=\min _{\Theta} \sum_{i=1} w_{i}\left(r^{i}\right)^{2},
$$

where $w_{i}=\frac{1}{r^{i}} \frac{\partial \Gamma}{\partial x}\left(r^{i}\right)$. The normal of the horizon plane has then the coordinates $(A, B, 1)$ and we can compute pitch $(\psi)$ and roll $(\rho)$ angles as follows :

$$
\begin{gathered}
\psi=\frac{A}{|A|} \times \arccos \left(\frac{1}{\sqrt{1+A^{2}}}\right) \\
\rho=\frac{-B}{|B|} \times \arccos \left(\frac{|\cos (-\psi)-A \sin (-\psi)|}{\sqrt{(\cos (-\psi)-A \sin (-\psi))^{2}+B^{2}}}\right)
\end{gathered}
$$

\section{EXPERIMENTAL RESULTS}

\section{A. Horizon Detection}

The results of the horizontal detection on three different sequences are presented on Figure 4. These images have been taken with our sensor from an altitude of 2 meters. In this figure, the white line corresponds to the limit between the sky and the ground. The minimization of equation(11) is performed with the HCF algorithm [15]. We see that Markovian modeling permits to correctly delimit the sky and the ground, even in the presence of trees (Fig 4(c) and Fig $4(d))$. On the other hand, when the texture of the ground is similar to that of the sky, the first one is labelled as belonging to the sky (Ex: snow Fig 4(e) and Fig 4(f)). In this case, the M-estimator plays an important role. Indeed, as long as the number of points which delimits this badly labelled zone is weak compared to the number of points which detect the horizon correctly, the M-estimator will not take into account these outliers.

\section{B. Attitude Computation}

Since it is difficult to obtain the ground truth for the attitude of a UAV synchronized with the correspondent image, we evaluate our algorithm with synthetic images. We then use a synthetic catadioptric image generator with the real intrinsic parameters of our catadioptric sensor. The altitude of the UAV is constant and equal to 500 meters. We can then easily compute the occluding contour of the Earth, and consequently its projection on the catadioptric image plane. 11,000 artificial images of the projection of the horizon were generated with different combinations of roll and pitch angles. In order to study the sensitivity of the algorithm to image noise, we add outliers to the list of points which constitute the projected 


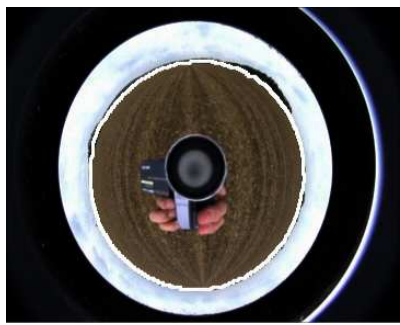

(a)

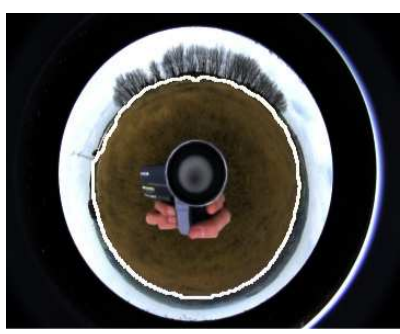

(c)

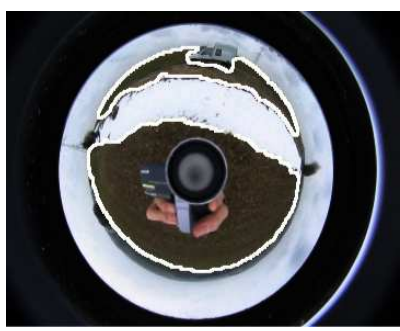

(e)

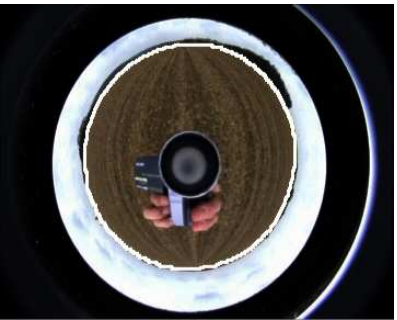

(b)

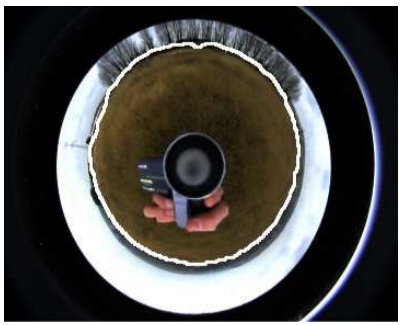

(d)

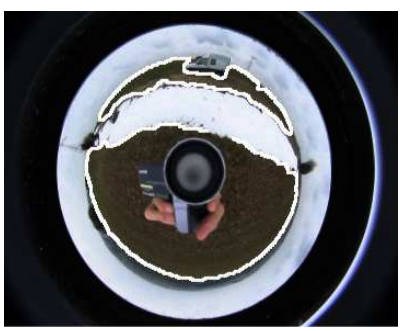

(f)
Fig. 4. Results of Sky/Ground Segmentation using the Markovian modeling with a neighborhood adapted for omnidirectional image, $\mu=10$.

horizon. The number of outliers varies from 0 to $100 \%$ of the number of horizon points and the positions of these outliers are randomly computed.

In Figure 5, the 3D plots show the mean error expressed in degrees for the roll and pitch angles for the different combinations of angles, noise and sampling. We can see that this error is approximately equal to 3 degrees when the number of outliers is low and increases to 5 degrees when the number of outliers is high. If we analyze our results more in depth, the main errors are obtained when the pitch and roll angles are close to $90^{\circ}$ or $-90^{\circ}$. If we consider only the interval equal to $\left[-60^{\circ}, 60^{\circ}\right]$ for both angles, the error becomes less than 1 degree. This effect is due to the projection of the horizon which becomes a piece of ellipse in the image. The estimation of the parameters of the great circle is then more delicate and some errors may occur.

In table I, we present the roll and pitch angles of Figure 5. For each one of these images, we do not have the ground truth attitude but we can estimate that the results are coherent with the presented images. Finally, in Figure 6, we propose some results obtained with the sensor mounted on an aerial platform. The red pixels correspond to the detected pixels of the horizon and in the bottom left corner, the estimated roll and pitch angles are presented. We can see that the evolution

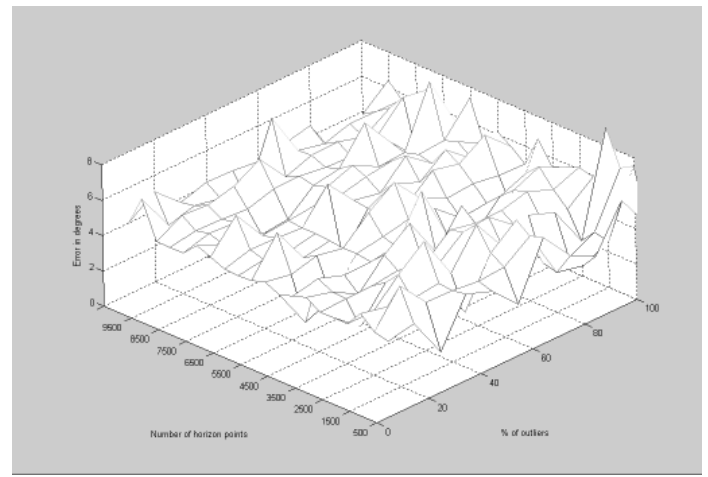

(a)

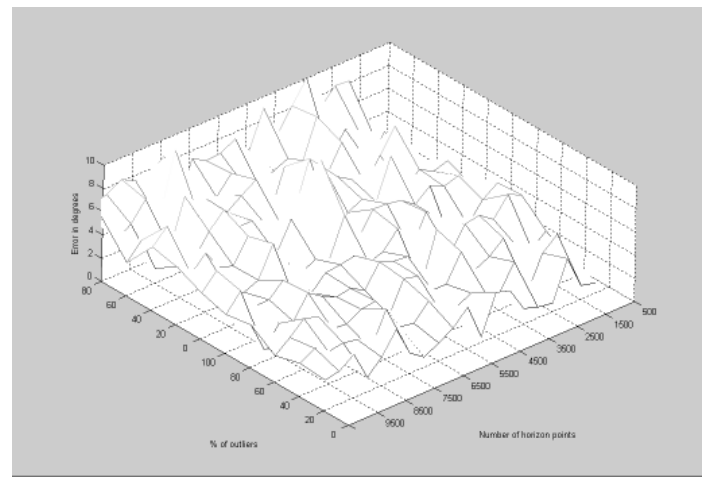

(b)

Fig. 5. 3D plot of the attitude error in the different combinations (a) Roll error, (b) Pitch error.

\begin{tabular}{|c|c|c|}
\hline Figure & Roll $(\rho)$ & Pitch $(\psi)$ \\
\hline 5.(a) & $\rho=-5.5^{\circ}$ & $\psi=-3^{\circ}$ \\
5.(b) & $\rho=2.3^{\circ}$ & $\psi=3.5^{\circ}$ \\
5.(c) & $\rho=-3^{\circ}$ & $\psi=-9^{\circ}$ \\
5.(d) & $\rho=-2.9^{\circ}$ & $\psi=5.2^{\circ}$ \\
5.(e) & $\rho=0^{\circ}$ & $\psi=-4^{\circ}$ \\
5.(f) & $\rho=-4.2^{\circ}$ & $\psi=-1.6^{\circ}$ \\
\hline
\end{tabular}

TABLE I

ROLL AND PITCH ANGLES OF FIGURE 5

of the values is totally coherent with the movement perceived in the sequence. The complete sequence is available at the following address http://www.crea.u-picardie.fr/ vasseur. Figure 7 presents the evolution of the roll and pitch angles during a sequence of 1000 images. We can note that for each image the angles are always computed and that there is no outlier in the estimated values. We can also see that the algorithm offers a good rate of repeatability since between successive images with a similar attitude, the angle values do not differ significantly.

\section{SUMmARY AND CONCLUSION}

In this paper, we present an attitude sensor for UAVs based on a central catadioptric vision sensor. This sensor presents several advantages compared to a perspective camera such as robustness even in case of occlusions in the horizon, the 


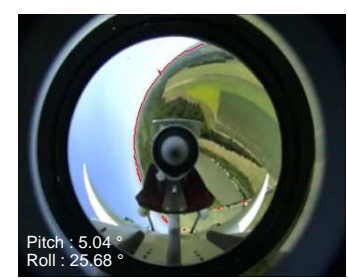

(a)

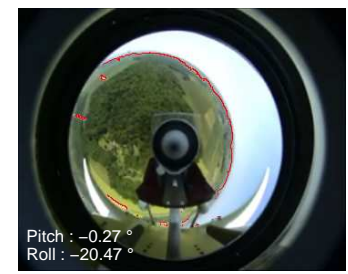

(c)

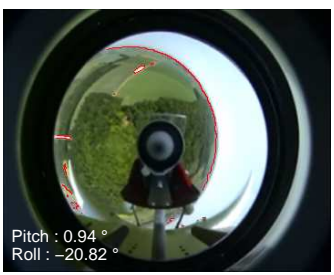

(e)

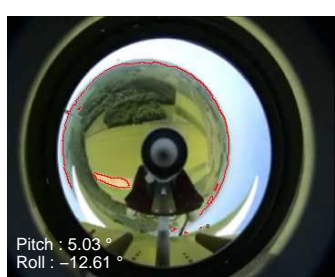

(b)

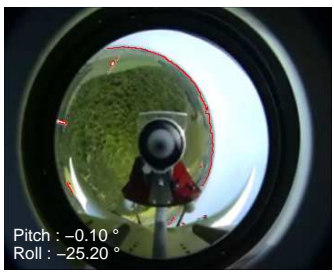

(d)

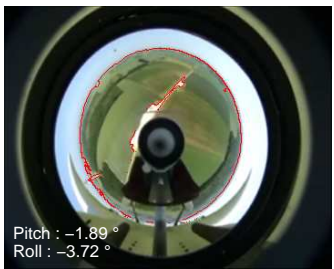

(f)
Fig. 6. Experimental results on real images captured from an aerial platform

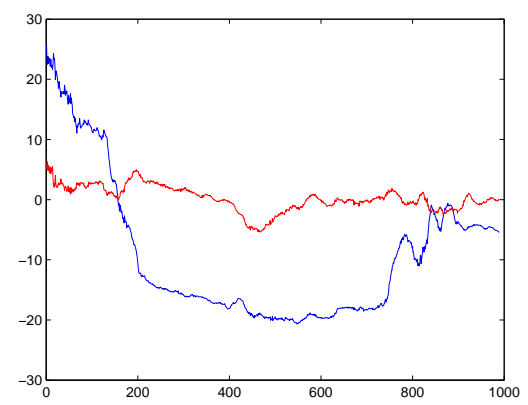

Fig. 7. Roll (blue line) and pitch (red line) angles during a sequence of 1000 images.

constant visibility of the horizon in the image and a real computation of the roll and pitch angles. The method for the attitude computation is divided in two main steps. First, we are looking for the horizon line in the catadioptric image plane. This detection is performed thanks to a markovian approach. However, because of the distortions in the omnidirectional image, a classic neighborhood can not be directly employed. We then propose a new neighboring system adapted to the geometry of the sensor in order to segment the image in two regions (sky/earth). Once the horizon line is detected, the second step consists in projecting this line onto the equivalent sphere in order to estimate the attitude angles. Thanks to the geometric properties of the equivalent sphere, the computation of the pitch and roll angles consists in finding a 3-space plane which intersects the sphere. For this, we use a robust estimator in order to discard the potential outliers detected during the horizon line extraction. Experimental results demonstrate the robustness of the horizon line detection and attitude estimation of the sensor (and consequently of the UAV). Synthetic results allow to verify the accuracy of the angle estimation with an error which is less than one degree for angles in the interval $\left[-60^{\circ}, 60^{\circ}\right]$.

\section{ACKNOWLEDGMENT}

This work is supported by Agence Nationale de la Recherche - projet CAVIAR (ANR-05-BLAN-0291-01).

\section{REFERENCES}

[1] S. Ettinger, M. Nechyba, P. Ifju, and M. Waszak, "Vision-guided flight stability and control for micro air vehicles," In Advanced Robotics, vol. 17 , no. 7, pp. 617-640, 2003.

[2] S. Todorovic, M. Nechyba, and P. Ifju, "Sky/ground modeling for autonomous mav flight," in In Proc. IEEE International Conference on Robotics and Automation (ICRA03), Taipei, Taiwan, May 2003, pp. 1422-1427.

[3] S. Hrabar and G. Sukhatme, "Omnidirectional vision for an autonomous helicopter," in In Proc. IEEE International Conference on Robotics and Automation (ICRA03), Taipei, Taiwan, May 2003, pp. 558-563.

[4] R. Benosman and S. Kang, Panoramic Vision: Sensors, Theory, Applications. Springer, 2001.

[5] K. Daniilidis, A. Makadia, and T. Blow, "Image processing in catadioptric planes: Spatiotemporal derivatives and optical flow computation." in In Proc. IEEE Workshop on Omnidirectional Vision (OMNIVISO2), Copenhagen, Denmark, June 2002, pp. 3-10.

[6] S. Baker and S. Nayar, "A theory of catadioptric image formation," in In Proc. of International Conference on Computer Vision (ICCV98), Bombay, India, January 1998, pp. 35-42.

[7] C. Geyer and K. Daniilidis, "Catadioptric projective geometry," Int. Journal Computer Vision, vol. 45, no. 3, pp. 223-243, 2001.

[8] J. Barreto and H. Araujo, "Issues on the geometry of central catadioptric image formation," in In Proc. Conference on Computer Vision and Pattern Recognition (CVPRO1), Kauai, HI, USA,, December 2001, pp. 422-427.

[9] X. Ying and Z. Hu, "A theory of catadioptric image formation," in In Proc. of International Conference on Computer Vision (ICCVO3), Nice, France, October 2003, pp. 1351-1358.

[10] J. Besag, "Spatial interaction and the statistical analysis of lattice systems," RoyalStat, vol. B-36, no. 2, pp. 192-236, 1974.

[11] C. Demonceaux and P. Vasseur, "Champs de markov pour le traitement d'images catadioptriques." Traitement du Signal, to be published.

[12] M. Swain and D. Ballard, "Color indexing," Int. Journal Computer Vision, vol. 7, no. 1, pp. 11-32, 1991.

[13] Z. Zhang, "Parameter estimation techniques: A tutorial with application to conic fitting," In Image and Vision Computing (IVC), vol. 15, pp. 59-76, 1997.

[14] P. Huber, Robust Statistics. J.Wiley and Sons, 1981.

[15] P. Chou and C. Brown, "The theory and practice of bayesian image labeling," Int. Journal Computer Vision, vol. 4, no. 3, pp. 185-210, 1990. 